\title{
Impact of Insurance Scheme on Adults' Dental Check-Ups in a Developing Oral Health Care System
}

Fariborz Bayat ${ }^{\mathrm{a}, \mathrm{b}}, \mathrm{DDS}$

Miira M Vehkalahtia, DDS, PhD

A. Hamid Zafarmand ${ }^{b}$, DDS, MS, PhD

Heikki Talaa, DDS, MPH, PhD

\section{ABSTRACT}

Objectives: To find out the relationship between dental insurance and demand for dental care, the present study evaluated impact of insurance scheme on adults' dental check-ups in a developing oral health care system.

Methods: The target population included adults in the city of Tehran where the only telecommunication company provides $90 \%$ of the 1.9 million households with a fixed telephone. Of the 1531 subjects who answered the phone call, 224 were outside the target age (under 18), 67 said that they never had visited a dentist, and 221 refused to respond, leaving 1019 subjects in the final sample. Each interview lasted 15 minutes and was carried out using a structured questionnaire with fixed and open-ended questions.

Results: $71 \%$ of the subjects reported having dental insurance and $16 \%$ having visited a dentist for a check-up; 55\%, more women than men, reported having had a dental visit within the past 12 months.

Conclusions: The present results revealed the positive relationship between insurance and demand for dental care. Those having dental insurance were more likely to go to check-ups despite their generally low rate found in this country with a developing oral health care system. In such countries, health insurance schemes should therefore include obligatory regular dental check-ups to emphasize prevention-oriented dental care. (Eur J Dent 2008;2:3-10)

Key words: Adults; Dental check-ups; Dental visit; Demand; Dental insurance.

\section{INTRODUCTION}

The reason to visit a dentist is regarded as one of the measures of demand since it is the users who determine the action. ${ }^{1}$ As reviewed by

a Institute of Dentistry, University of Helsinki, Helsinki, Finland

b Shaheed Beheshti Medical University, Tehran, Iran

Corresponding author: Dr. Fariborz Bayat Dept. of Oral Public Health Institute of Dentistry, P.O.Box: 41 University of Helsinki FI-00014 Helsinki Finland

Tel: +358-9-19127301

Fax: +358-9-191 27346

E-mail: fariborz.bayatahelsinki.fi
Bendall, ${ }^{2}$ dental insurance is positively related to the demand for dental care. In comparison with the non-insured subjects, those with dental insurance visit a dentist more regularly ${ }^{3-6}$ and have frequent dental check-ups. ${ }^{7-10}$

As part of preventive health behavior, ${ }^{11}$ dental check-ups are commonly recommended. ${ }^{12-15}$ Generally, health behavior is affected by individual characteristics, such as age, gender, and level of education but also by the characteristics of the oral health care system, such as availability of third party payment options. ${ }^{16}$ Depending on the social and political system of each country, 
the health policy, oral health services, and their funding can support preventive oral health behavior in populations by encouraging checkups, emphasizing preventative orientation of the dental services, and applying third party payment systems.

In the pure model of secondary prevention, regular check-ups have two purposes: to reduce the burden of illness by accurate detection of disease at its early stage and to treat the disease effectively in order to alter its natural course or progression. ${ }^{17-}$ 18 Policy in most of the Scandinavian countries, in the UK, and the Netherlands emphasizes regular dental check-ups for all.6,8,20,22-23 In developed countries, the rates of annual check-ups for adults range from $54 \%$ to $92 \%,{ }^{20,22-25}$ but even higher rates of dental check-ups have been reported for people with private dental insurance..$^{8-10,24}$

In many developing countries, dental care systems focus on treatment and relief of pain. ${ }^{25-28}$ Treatment-oriented national insurance systems may discourage preventive use of oral health services. ${ }^{29}$ Thus, patterns of adults' behavior for dental check-ups according to insurance status may differ from those in developed countries with prevention-oriented health insurance programs.

To find out the relationship between dental insurance and demand for dental care, the present study evaluated impact of insurance scheme on adults' dental check-ups in a developing oral health care system.

\section{MATERIALS AND METHODS}

\section{Background}

Table 1 describes the health insurance system in Iran. The main function of both public and commercial insurance is to cover the costs of treatments; neither scheme has any obligation to attend dental check-ups. Public insurance covers $50 \%$ of Iran's population, and commercial insurance almost $10 \%$ of the employees. ${ }^{30}$ The dentist-population ratio in Tehran is 1:1800, with around 1300 dentists who are hired by or have contracted with insurance schemes. ${ }^{30-33}$

\section{Design and sampling}

The Iranian Centre for Dental Research granted ethical approval for the present study, which was based on cross-sectional data obtained through phone interviews. The target population included adults in the city of Tehran where the only telecommunication company provides $90 \%$ of the 1.9 million households with a fixed telephone. A pilot study in February 2005 on the sampling method showed its feasibility. Then, a total of 3200 seven-digit numbers were randomly selected from a list of four million computerized options resembling real phone numbers.

\section{Phone calls}

Four trained interviewers made the phone calls. The outcome of each successful call was recorded as the duration of the interview and of a missed call as the reason for failure (busy, no answer, fax, and non-connected lines). After five attempts, a busy or non-answering line was omitted from the list. In total, 1669 phone numbers were unavailable, most because of being for a non-connected line or fax.

\section{Interviewing and questions}

Each interview lasted 15 minutes and was carried out using a structured questionnaire with fixed and open-ended questions. The questions were based on related recent studies. ${ }^{29,34-35}$ The first question asked for the respondent's date of birth, later calculated as the respondent's age to the nearest year. The answers to the question about insurance status were recorded as: No insurance, Public insurance, or Commercial insurance.

For the question "What was the reason for your last dental visit?", the answer was recorded as: Check-up, Trouble with teeth or gums, Continuing dental treatment, and Never visited a dentist. In the analyses, the very rare cases who responded that they were continuing their treatment (mostly a root canal treatment or fixed prosthetics) were combined into the category of Trouble with teeth or gums. Those who said that they had never visited a dentist were excluded. Consequently, the reason for the dental visit was dichotomized as Check-up or Trouble.

Utilization of dental services was inquired as the number of dental visits within past 12 months and was recorded as: No visit, One, Two, Three, Four and more, and I do not remember. For the analysis, the latter option with very rare cases $(1.3 \%)$ was taken as No dental visit. For the crosstabulation, number of dental visits was categorized 
as: None, One, Two and more.

The respondent's level of education was recorded using six options, later combined into three: Low lilliterate, primary or secondary school), Medium (high school or diploma), and High lassociate degree, bachelor's degree, master degree). Information about family income was obtained with the open question "How much is your monthly household income?" the answers were recorded as an amount of Rials and then categorized into Low (under the poverty line), Medium, and High.

\section{Statistical analysis}

Evaluation of the statistical significance of the differences between the subgroups included the Chi-squared test for frequencies and ANOVA for comparison of mean values. The strength of the factors related to a dental check-up visit for was evaluated by fitting a logistic regression model to the data and by calculating the corresponding odds ratios (OR) and their $95 \%$ confidence intervals (95\% CI). Goodness of fit was evaluated by means of the Hosmer and Lemeshow test.

Of the 1531 subjects who answered the phone call, 224 were outside the target age (under 18), 67 said that they never had visited a dentist, and 221 refused to respond, leaving 1019 subjects in the final sample. Their age pattern approximates the age distribution of the target population. ${ }^{36}$

\section{RESULTS}

Of the subjects ( $n=1019$ ), $58 \%$ were women and $61 \%$ were under 35 years of age. The distribution by age was similar for men and women ( $P=0.28)$. Subjects' mean age was 32.9 years $(S D=10.7$; median 32.0: 95\% Cl=32.2-33.5), with no gender differences ( $P=0.18)$. Of all, $71 \%$ reported having dental insurance and $16 \%$ having visited a dentist for a check-up; 55\%, more women than men, reported having had a dental visit within the past 12 months.

Table 2 illustrates the rates of respondents reporting check-up as the reason for their most recent dental visit, and having two and more dental visits within last 12 months, according to their characteristics. The highest rates for checkups occurred in the youngest age groups: $24 \%$ for men $(P<.001)$ and $22 \%$ for women $(P=0.02)$, among those with a higher or medium level of education $(P<.001)$, and those with commercial insurance $(P<.01)$. Household income showed no impact on reporting dental check-ups.

With no gender difference, $30 \%$ of the respondents reported having had two and more visits within the past 12 months. That was most common among 25- to 34-year-olds $(P<.001)$, among those with high level of education and income $(P<.001)$, and among those with commercial insurance $(P=0.03)$

Table 1. Description of dental insurance system in Iran.

\begin{tabular}{|c|c|c|}
\hline Aspects of insurance & Public insurance & Commercial insurance \\
\hline Funding & $\begin{array}{l}\text { Premium: } 70 \% \text { by the employer, } 30 \% \\
\text { by the employee }\end{array}$ & $\begin{array}{l}\text { Premium: by the employer as fringe } \\
\text { benefits }\end{array}$ \\
\hline Reimbursement to dentist & Monthly salary & $\begin{array}{l}\text { Fee-for-service payment under a fixed- } \\
\text { fee quotation }\end{array}$ \\
\hline Access and restrictions & $\begin{array}{c}\text { Available at the clinics owned ( } n=19) \\
\text { by or contracted ( } n=160) \text { with public } \\
\text { insurance. } \\
\text { Restricted to basic dental services. }\end{array}$ & $\begin{array}{l}\text { Available at clinics contracted with } \\
\text { commercial insurance }(n=300) \text {. } \\
\text { No restriction by type of dental services. } \\
\text { Annual ceiling for the reimbursement. }\end{array}$ \\
\hline Target population & $\begin{array}{l}\text { Obligatory for all employees (and their } \\
\text { family members) under the labour act. }\end{array}$ & $\begin{array}{l}\text { Employees (and their family members) of } \\
\text { commercial enterprises. }\end{array}$ \\
\hline Population coverage & About $50 \%$ & Less than $10 \%$ \\
\hline Dentist-population ratio ${ }^{1}$ & $1: 3000$ & $1: 1100$ \\
\hline
\end{tabular}

Estimates based on data provided by statistical center of Iran (1), Social security (2) and, Medical service insurance organization (3), and Medical council of Iran (4). 
Among those subjects who had at least one dental visit within the past 12 months, number of visits was slightly higher for women than men (2.1 v.s. 2.0; $P=0.05$ ). Table 3 shows mean numbers of dental visits according to the respondents' characteristics. Higher numbers of dental visits were found for those with a high level of education $(P<.001)$ and those with commercial insurance $(P=0.05)$.

In a multivariable analysis household income as a co-variable revealed no impact and was omitted from the further analysis. The level of education, the type of insurance, and number of dental visits appeared as the main explanatory factors for subjects' dental check-ups in the final logistic regression analysis (Table 4), which simultaneously controls for all factors included. The model indicated that those with a medium (OR=2.6) or high (OR=3.3) level of education, and with commercial insurance (OR=2.4) were more likely to go to a dentist for a check-up. The model fitted the data well $(P=0.62)$.

\section{DISCUSSION}

Only $16 \%$ of our respondents gave a check-up as the reason for their most recent dental visit. In comparison with developed countries, this is far from the recommended way to use dental services. In Netherlands, almost all insured patients (92\%), both public and private, reported that they had

Table 2. Percentages of adults (1019) reporting a check-up as the reason for their most recent dental visit and having had at least two visits within the past 12 months according to their characteristics, separately for men ( $\mathrm{n}=429$ ) and women ( $\mathrm{n}=590)$, in Tehran, Iran.

\begin{tabular}{|c|c|c|c|c|c|c|c|}
\hline \multirow{2}{*}{\multicolumn{2}{|c|}{ Characteristics }} & \multicolumn{3}{|c|}{ Check-ups (\%) } & \multicolumn{3}{|c|}{$\geq 2$ dental visits (\%) } \\
\hline & & Men & Women & All & Men & Women & All \\
\hline \multicolumn{8}{|l|}{ Age group } \\
\hline & $18-24$ & 24 & 22 & 23 & 24 & 34 & 29 \\
\hline & $25-34$ & 16 & 19 & 18 & 34 & 33 & 33 \\
\hline & $35-44$ & 9 & 11 & 10 & 28 & 32 & 30 \\
\hline & $45+$ & 4 & 11 & 8 & 14 & 31 & 24 \\
\hline & $P$ value & $<0.001$ & 0.02 & $<0.001$ & $<0.001$ & 0.52 & $<0.001$ \\
\hline \multicolumn{8}{|c|}{ Level of education } \\
\hline & Low & 5 & 6 & 5 & 12 & 19 & 17 \\
\hline & Medium & 17 & 17 & 17 & 23 & 32 & 28 \\
\hline & High & 17 & 23 & 20 & 38 & 42 & 40 \\
\hline & $P$ value & 0.03 & $<0.001$ & $<0.001$ & $<0.001$ & $<0.001$ & $<0.001$ \\
\hline \multicolumn{8}{|c|}{ Household income ${ }^{1}$} \\
\hline & Low & 4 & 16 & 11 & 17 & 30 & 25 \\
\hline & Medium & 16 & 14 & 15 & 27 & 31 & 29 \\
\hline & High & 18 & 19 & 18 & 32 & 44 & 38 \\
\hline & $P$ value & 0.08 & 0.54 & 0.21 & 0.20 & $<0.001$ & $<0.001$ \\
\hline \multicolumn{8}{|c|}{ Dental insurance status } \\
\hline & No-insurance & 12 & 14 & 13 & 23 & 26 & 25 \\
\hline & Public insurance & 16 & 16 & 16 & 28 & 34 & 31 \\
\hline & Commercial insurance & 25 & 31 & 28 & 36 & 47 & 42 \\
\hline & $P$ value & 0.17 & 0.05 & 0.01 & 0.66 & 0.05 & 0.03 \\
\hline
\end{tabular}

\footnotetext{
${ }^{1}$ No answer by 269 respondents.
} 
visited a dentist for a check-up within the past 12 months. ${ }^{20}$ High or moderate check-up rates have been reported for the USA, 78\%, ${ }^{8}$ Finland, 57\%, ${ }^{35}$ Australia, $53 \%{ }^{37}$ and Japan, $46 \% .^{13}$ In the UK, $62 \%$ of adults report having had a dental check-up within the previous 12 months, the figures being clearly higher for those under the NHS (46\%) compared to $14 \%$ for the non-NHS subjects. ${ }^{38}$

The behavior of visiting a dentist regularly for check-ups has its origins in one's childhood. In addition, the health policy and the characteristics of the oral health care system in a community create and maintain circumstances favorable to such behavior. One important and effective way to promote this behavior has been schoolbased dental care, where children visit a dentist for check-ups at regular intervals. Studies have shown that this preventive behavior seems to continue into adulthood. ${ }^{29,39-40}$ Consequently, in those countries with higher rates for dental check-ups, school-based dental care programs have long dominated. ${ }^{41}$ In Iran, the public health services offer dental care to school children up to 12 years of age. ${ }^{42}$ The fact that this care does not include regular dental check-ups is probably reflected in the present adults' check-up behavior as well.

Those insurance health systems with prevention-oriented features and an obligation to regular dental check-ups have resulted into higher rates of check-ups. ${ }^{7}$ The very low rates of checkups in the present study certainly reflect the nature of the health delivery system. Unfortunately, Iran has a treatment-oriented health care system where patients usually make a dental visit when they have trouble with their teeth or gums. The policies of either public or commercial insurance include no obligation to attend regular dental check-ups.

In our study, having a commercial insurance had a strong impact on attendance at dental checkups. This is in line with findings from countries with predominantly private insurance schemes and high rates of dental check-ups..$^{7-10,22,47}$ In Iran, commercial insurance is employer-sponsored, and dental services are offered to employees and their families as fringe benefits. These benefits are supposed to be used annually. For our adults, the ceiling of the annual remuneration by the insurance seemed to have led to symptom-based care rather than to regular dental check-ups.

The strong relationships we observed between

Table 3. Mean number of dental visits among those adults who have at least one dental visit within last 12 months ( $n=562)$, separately for men ( $n=225$ ) and women ( $=337)$.

\begin{tabular}{|c|c|c|c|c|}
\hline \multirow{3}{*}{ Characteristics } & \multicolumn{4}{|c|}{ Mean number of visits } \\
\hline & All & Men & Women & \\
\hline & Mean (SD) & Mean (SD) & Mean (SD) & $P$ value \\
\hline \multicolumn{5}{|l|}{ Age group } \\
\hline $18-24$ & $2.0(1.2)$ & $1.8(1.1)$ & $2.1(1.2)$ & \multirow{4}{*}{0.65} \\
\hline $25-34$ & $2.1(1.2)$ & $2.0(1.1)$ & $2.2(1.2)$ & \\
\hline $35-44$ & $2.2(1.2)$ & $2.2(1.2)$ & $2.2(1.2)$ & \\
\hline $45+$ & $2.1(1.1)$ & $1.7(1.0)$ & $2.2(1.2)$ & \\
\hline \multicolumn{5}{|l|}{ Level of education } \\
\hline Low & $1.8(1.1)$ & $1.6(1.1)$ & $1.9(1.2)$ & \multirow{3}{*}{$<0.001$} \\
\hline Medium & $2.0(1.2)$ & $1.8(1.0)$ & $2.2(1.2)$ & \\
\hline High & $2.3(1.2)$ & $2.3(1.1)$ & $2.3(1.2)$ & \\
\hline \multicolumn{5}{|l|}{ Household income ${ }^{1}$} \\
\hline Low & $2.2(1.2)$ & $1.2(1.1)$ & $2.3(1.2)$ & \multirow{3}{*}{0.80} \\
\hline Medium & $2.0(1.2)$ & $2.0(1.1)$ & $2.1(1.2)$ & \\
\hline High & $2.1(1.2)$ & $2.0(1.1)$ & $2.3(1.2)$ & \\
\hline \multicolumn{5}{|l|}{ Dental insurance status } \\
\hline No-insurance & $2.0(1.1)$ & $1.8(1.0)$ & $2.0(1.2)$ & \multirow{3}{*}{0.05} \\
\hline Public insurance & $2.1(1.2)$ & $2.0(1.1)$ & $2.1(1.2)$ & \\
\hline Commercial insurance & $2.2(1.2)$ & $2.1(1.1)$ & $2.3(1.2)$ & \\
\hline
\end{tabular}

Statistical evaluation for comparison of mean values by ANOVA.

${ }^{1}$ No answer by 139 respondents 
dental check-ups and levels of education have also been found by several other studies. In Iran, those who have a high or medium level of education are usually employed, and they also tend to have higher income. That factor again, by itself, is associated with higher demand for dental care. In addition, people with higher education and income are more likely to have a dental insurance as a benefit.

Although recommendations regarding optimal recall intervals vary between countries and dental health care systems, general dental practitioners in many developed countries have traditionally advocated six-monthly dental check-ups. ${ }^{14}$ It can be assumed that rather many people with a high or medium level of education value their oral health and would follow dentist recommendations on regular check-ups. Based on the present low rates for check-ups, it seems that Iranian dentists have not adopted recalls in their everyday practice.

Disparities in the rates of dental check-ups according to gender and age were observed in the bivariate analyses, but these differences disappeared when controlled for in the logistic regression analyses. That gender was not correlated with dental check-up rates is an interesting finding in view of the frequent reports of higher demand for dental care among women. ${ }^{13,20,22,37,38}$ However, in our study population, more women than men reported a dental visit within the past 12 months.

In the present study, variation in numbers of dental visits within the past 12 months showed that level of education is associated with utilization. A wider evaluation of factors related to utilization of dental services could benefit of data of expenditures of oral health care. Unfortunately such information is not available for Iran. Nevertheless, the total expenditures of health in Iran are $6.6 \%$ of GDP (6.0\% in 2001).43 In addition, according to the Iran National Health Account, $18 \%$ of health expenditures are paid by health insurance, $23 \%$ by government and the rest is paid out-of-pocket. ${ }^{44}$

In the present study, convenience of the interviewers and respondents and access to the vast majority of the target population (90\%) were the important aspects for selecting the phone interview method. In addition, that is a costefficient method of data collection in area where the telephone system is well developed with a high coverage and cheap calls. Validity of such method for general and oral health research has been verified in several studies. ${ }^{37,45-46}$ Although, there is a possibility that, those unable to be contacted because of mobility or lack of telephone line may differ from the respondents in their use of dental services.

Table 4. Factors related to reporting that a check-up was the reason for most recent dental visit, as explained by means of a logistic regression model fitted to the data on adults reporting a dental visit ( $\mathrm{n}=1019)$ in Tehran, Iran.

\begin{tabular}{|c|c|c|c|c|c|c|}
\hline Parameters & & ES* & SE & $\mathrm{P}$ & OR & $\mathrm{Cl} 95 \%$ \\
\hline \multirow{5}{*}{ Level of education } & Age & -0.04 & 0.01 & $<0.001$ & 1.0 & $0.9-1.0$ \\
\hline & Gender & 0.17 & 0.18 & 0.35 & 1.2 & $0.8-1.7$ \\
\hline & & & & & & \\
\hline & Low & & & & 1.0 & \\
\hline & Medium & 0.94 & 0.36 & $<0.001$ & 2.6 & $1.3-5.2$ \\
\hline \multirow{3}{*}{ Type of insurance } & High & 1.20 & 0.36 & $<0.001$ & 3.3 & $1.6-6.7$ \\
\hline & & & & & & \\
\hline & Non- insured & & & & 1.0 & \\
\hline \multirow{4}{*}{$\begin{array}{l}\text { Dental visit within last } 12 \\
\text { months }\end{array}$} & Public insurance & 0.22 & 0.21 & 0.37 & 1.3 & $0.8-1.9$ \\
\hline & Commercial insurance & 0.90 & 0.35 & 0.01 & 2.4 & $1.3-4.7$ \\
\hline & No & & & & 1.0 & \\
\hline & Yes & 0.40 & 0.18 & 0.05 & 1.4 & $1.0-2.0$ \\
\hline
\end{tabular}

Goodness-of-fit by Hosmer and Lemeshow test. $p=0.62$

*Estimate of strength 


\section{CONCLUSIONS}

The present results revealed the positive relationship between insurance and demand for dental care. Those having dental insurance were more likely to go to check-ups despite their generally low rate found in this country with a developing oral health care system. In such countries, health insurance schemes should therefore include obligatory regular dental checkups to emphasize prevention-oriented dental care.

\section{ACKNOWLEDGMENTS}

We wish to thank all respondents for their participation.

\section{REFERENCES}

1. So FHC, Schwarz E. Demand for and utilization of dental services among Hong Kong employees with and without dental benefit coverage. Community Dent Oral Epidemiol 1996;24:201-206.

2. Bendall $D$, Asubonteng $P$. The effect of dental insurance on the demand for dental services in the USA: a review. $J$ Manag Med 1995;9:55-68.

3. U.S Department of Health and Human Services. Healthy people 2010. Understanding and improving health. Washington: U.S.Department of Health and Human Services, 2000.

4. Manning WG, Bailit HL, Benjamin B, et al. The demand for dental care: evidence from a randomised trial in health insurance. J Am Dent Assoc 1985;110:895-902.

5. Brennan DS, Carter K, Stewart J, et al. Commonwealth Dental Health Program Evaluation Report 1994-1996, Adelaide: AlHW Dental statistics and Research unite; Page 94-96.

6. Locker D, Leake JL. Inequities in health: dental insurance coverage and use of dental services among older Ontario adults. Can J Public Health 1993;84:139-140.

7. Sohn W, Ismail Al. Regular dental visits and dental anxiety in an adult dentate population. J Am Dent Assoc 2005;136:58-66.

8. Woolfolk MW, Lang WP, Borgnakke WS et al. Determining dental checkups frequency. J Am Dent Assoc 1999;130:715723.

9. Newman JF, Gift HC. Regular pattern of preventive dental services: a measure of access. Soc Sci Med 1992;35:9971001.

10. Lang WP, Farghaly MM, Ronis DL. The relation of preventive dental behaviors to periodontal health status. $J$ Clin Periodontol 1994:21:194-198.

11. Hayward RA, Meetz HK, Shapiro MF, Freeman HE.
Utilization of dental services: 1986 patterns and trends. $J$ Public Health Dent 1989;49:147-152.

12. Helminen SK, Vehkalahti MM. Do check-up intervals correspond to caries indices in the free public dental service in Helsinki, Finland? Community Dent Health 2002;19: 166-172.

13. Tamaki Y, Nomura Y, Teraoka K, Nishikahara F, Motegi M, Tsurumoto A, Hanada N. Characteristics and willingness of patients to pay for regular dental check-ups in Japan. $J$ Oral Sci 2004:46:127-133.

14. Beirne P, Forgie A, Clarkson J, Worthington HV. Recall intervals for oral health in primary care patients. Cochrane Database Syst Rev 2005;18:CD004346.

15. Mettes TG, van der Sanden WJ, Mulder J, Wensing M, Grol RP, Plasschaert AJ. Predictors of recall assignment decisions by general dental practitioners performing routine oral examinations. Eur J Oral Sci 2006;114:396-402.

16. Kiyak HA. Age and culture: influences on oral health behaviour. Int Dent $J$ 1993;43:9-16.

17. Perlus J. Determining recall frequency; A controversial issue. Ontario Dentist 1994;71:31-35.

18. Petersen PE, Holst D. Utilization of dental health services. In: Cohen LK, Gift HC (eds) Disease prevention and oral health promotion: Socio-dental sciences in action. pp 34186. Copenhagen, Denmark: Munksgaard International Publisher, 1995

19. Abraham D. How dental utilization in The Netherlands was affected by a radical reform of the dental insurance system. Community Dent Health 2003;20:34-39.

20. Schouten BC, Mettes TG, Weeda W, Hoogstraten J. Dental check-up frequency: preferences of Dutch patients. Community Dent Health 2006;23:133-139.

21. Schwarz E, Kronborg D. Utilization of alternative delivery programs in youth dental care in Denmark. Community Dent Oral Epidemiol 1988;16:336-340.

22. Grytten J. Competition and dental services. Health Econ 2000;9:447-461.

23. Nuttall NM, Bradnock G, White D, Morris J, Nunn J. Dental attendance in 1998 and implication for future. Br Dent $J$ 2001;190:177-182.

24. Ronis DL, Lang WP, Farghaly MM, Passow E. Tooth brushing, flossing, and preventive dental visits by Detroit-area residents in relation to demographic and socioeconomic factors. J Public Health Dent 1993;53:138-145.

25. Zhu L. Oral health knowledge, attitudes and behaviour of adults in China. Int Dent $J$ 2005;55:231-241.

26. Zavras D, Economou C, Kyriopoulos J. Factors influencing dental utilisation in Greece. Community Dent Health 2004:21:181-188

27. Matee M, Simon E. Utilization of dental services in Tanzania 
before and after the introduction of cost-sharing. Int Dent $J$ 2000;50:69-72.

28. Lo EC, Lin HC, Wang ZJ. Utilization of dental services in Southern China. J Dent Res 2001;80:1471-1474.

29. Chen M, Anderson RM, Barmes DE et al. Comparing oral health care systems; a second international collaborative study. Geneva: World Health Organization 1997.

30. Iran statistical yearbook. Tehran: Statistical Centre of Iran. 2003. Available at: http://www.sci.org.ir/farsi/default.htm.

31. Social Security Organization. Cure Deputy of SSO, list of contracted dentist. Available at: www2.darman.sso.ir

32. Medical Service Insurance Organization. Contracted health care centers, List of contracted clinics and dentists. Available at: http://msio.org.ir

33. Medical Council of Iran. Available at: www.irimc.org

34. Tseveenjav B. Preventive dentistry in Mongolia. Academic dissertation. Helsinki: University of Helsinki, 2004. Availableat:http://ethesis.helsinki.fi/julkaisut/laa/hamma/ vk/tseveenjav.

35. Suominen-Taipale A. Demand for oral health care services in adult Finns. Academic dissertation. Turku: University of Turku, 2000.

36. Bayat F, Vehkalahti M.M, Tala H, Zafarmand HA. Dental attendance by insurance status among adults in Tehran, Iran. Int Dent J 2006;56:338-344.

37. Carter KD. Stewart JF. National Telephone Interview Survey 2002. AlHW Cat No 128. Adelaid: AIHW Dental Statistics and Research Unit, the University of Adelaide 2003.

38. Batchelor PA. Changes in self-reported attendance of British adults for dental check-ups between 1991 and 2000. Prim Dent Care 2004;11:125-130.

39. Schwarz E. Changes in demand for dental care among Danish adults, 1975-90. Acta Odontol Scand 1996;54:36-43.

40. Anderson R, Marcus M, Mahshigan M. A comparative systems perspective on oral health promotion and disease prevention. In Cohen LK, and Gift HC (eds) Oral Health Promotion: Socio-dental Sciences in Action. pp 307-340. Copenhagen: Munksgaard International Publishers 1995.

41. Birch S, Anderson R. Financing and delivering Oral Health Care: what can we learn from other countries? J Can Dent Assoc 2005;71:243-243d.

42. Pakshir HR. Oral health in Iran. Int Dent $J$ 2004;54:367372.

43. WHO statistical information system. Total expenditure on health as percentage of gross domestic product, 2004 Available at: http://www.who.int/countries/irn/en/

44. Aljunid SM. Health financing in Iran, Source of funding for health financing in Iran 1980-2001, World Health Organization, Eastern Mediterranean Region Iran Country Office.
45. Douglas E. Validity of Telephone Interview Data for Vascular Disease Risk Factors in a Racially Mixed Urban Community: The Northern Manhattan Stroke Study Neuroepidemiology 1999;18:174-184.

46. Ezzati-Rice T, Cynamon M, Blumberg S, et al. Use of an existing sampling frame to collection broad based health and health related data at the state and local level. National center for health statistics 1997. available at; www.cdc.gov/nchs/data/slaits/slaits_tele_survey.pdf 